\title{
Floquet Graphene Antidot Lattices
}

\author{
Andrew Cupo, ${ }^{1,}$ | Emilio Cobanera, ${ }^{2,1}$ James D. Whitfield, ${ }^{1}$ Chandrasekhar Ramanathan, ${ }^{1}$ and Lorenza Viola ${ }^{1,}$, $^{\dagger}$ \\ ${ }^{1}$ Department of Physics and Astronomy, Dartmouth College, Hanover, New Hampshire, 03755, USA \\ ${ }^{2}$ Department of Physics, SUNY Polytechnic Institute, Utica, New York, 13502, USA
}

\begin{abstract}
We establish the theoretical foundation of the Floquet graphene antidot lattice, whereby massless Dirac fermions are driven periodically by a circularly polarized electromagnetic field, while having their motion excluded from an array of nanoholes. The properties of interest are encoded in the quasienergy spectra, which are computed non-perturbatively within the Floquet formalism. We find that a rich Floquet phase diagram emerges as the amplitude of the drive field is varied. Notably, the Dirac dispersion can be restored in real time relative to the gapped equilibrium state, which may enable the creation of an optoelectronic switch or a dynamically tunable electronic waveguide. As the amplitude is increased, the ability to shift the quasienergy gap between high-symmetry points can change which crystal momenta dominate in the scattering processes relevant to electronic transport and optical emission. Furthermore, the bands can be flattened near the $\Gamma$ point, which is indicative of selective dynamical localization. Lastly, quadratic and linear dispersions emerge in orthogonal directions at the $M$ point, signaling a Floquet semi-Dirac material. Importantly, all our predictions are valid for experimentally accessible near-IR radiation, which corresponds to the above bandwidth limit for the graphene antidot lattice. Cycling between engineered Floquet electronic phases may play a key role in the development of next-generation on-chip devices for optoelectronic applications.
\end{abstract}

\section{INTRODUCTION}

Ever since graphene was fully isolated and characterized in 2004, it has remained the platform of choice for investigating massless Dirac fermions in two space dimensions (2D) [1. Graphene is a strong 2D material that can be easily and stably shaped into, for example, nanoribbons. The interplay between confinement and quantum effects (in the following, quantum confinement for short) opens an electronic band gap that increases with decreasing width 2 . Other experimentally accessible graphene nanostructures, and the focus of this paper, are the antidot lattices of Ref.3. These structures are fabricated by patterning a periodic array of nanoholes into a graphene monolayer. Comparable to varying the width of a nanoribbon, varying the size of the supercell and/or the diameter of the holes of the graphene antidot lattice amounts to controlling quantum confinement and the electronic band gap. Subsequent work has showed that the shape of the holes, edge termination, and edge magnetism can have a profound effect on the way that the energy gap scales with the geometric parameters of the antidots [4]23. These structures can be fabricated by lithographic methods [24, 25].

In addition to geometric control, the electronic properties of a material can be modified by time-periodic driving via an electromagnetic field. As coherent-control capabilities have continued to advance in different experimental platforms, the physics of periodically driven "Floquet quantum matter" has attracted growing attention across quantum science. While the subject has been reviewed extensively elsewhere [26-32], here we only high-

\footnotetext{
*andrew.cupo@dartmouth.edu

†
}

light a few results for graphene. One can open and control gaps in the quasienergy spectrum by adjusting the polarization, photon energy, and electric field amplitude [33 41. It is also possible to induce non-trivial topology in "bulk" graphene with time-reversal symmetrybreaking circularly polarized light. As a consequence, chiral edge states appear for the corresponding system with open boundaries 4248 , which are predicted to be detectable spectroscopically [49 51]. The dynamical generation of non-trivial gapped topology explains the observation of the Floquet anomalous quantum Hall effect in graphene [52, 53].

In this paper we investigate the interplay between the spatial complexity of the graphene antidot lattice and a periodic driving force, and show that it leads to Floquet electronic phases beyond what is possible with standard graphene. A main motivation for our analysis stems from the fact that, for standard graphene, the above bandwidth limit can only be reached with extreme UV radiation, which is ionizing and not practical to produce at high intensities. By contrast, for the graphene antidot lattice we find that the same limit corresponds to experimentally realizable infrared (IR) photon energies. Moreover, while nanoribbons and quantum dots are also viable platforms for generating geometrically complex electronic states, the antidot lattice is the only one that is spatially extended in 2D.

We follow a well established approach for modeling the electronic structure of the graphene antidot lattices. Our starting point is the 2D massless Dirac Hamiltonian (Sec.II) to which we add a circularly polarized electromagnetic field within the minimal coupling scheme. The properties of the periodically driven system are characterized by quasienergy spectra, computed from the extended space formulation of the Floquet formalism (Sec.III). By varying the electric field amplitude on a fine grid for a fixed photon energy, we identify several 
parameter regimes where the quasienergy bands take on interesting characteristics (Sec. IV). Building on these characteristics, we identify three potential quantum engineering applications: a low-wavelength-pass electronic filter (Sec. IV), an optoelectronic switch, and a dynamically tunable electronic waveguide (Sec.V). We conclude in Sec.VI with a summary and outlook.

\section{BACKGROUND: GRAPHENE ANTIDOT LATTICES}

A graphene antidot lattice is the system that results from etching a periodic array of holes ("antidots") into a sheet of monolayer graphene. The holes should not be so large or packed so close together that they compromise the integrity of the free standing sheet. In this paper we will rely on the effective model of the graphene antidot lattices introduced and investigated in Refs. 3, 8, and 20. The starting line is the effective $2 \mathrm{D}$ massless Dirac fermion Hamiltonian for graphene,

$$
H_{\text {Dirac }}=v_{F} \boldsymbol{p} \cdot \boldsymbol{\sigma} \text {. }
$$

Here $v_{F}$ is the Fermi velocity, $\boldsymbol{p}$ is the momentum operator, and $\boldsymbol{\sigma}=\left[\sigma_{x}, \sigma_{y}\right]$ is the vector of $x$ and $y$ Pauli spin matrices. The internal degree of freedom is due to pseudo-spin, not the physical spin angular momentum degree of freedom, which is not included in this description. The Fermi velocity is calculated to be $v_{F}=$ $3 \tau d /(2 \hbar)$, where $\tau=2.7 \mathrm{eV}$ is the nearest-neighbor hopping parameter and $d=0.142 \mathrm{~nm}$ is the carbon-carbon bond length. We note that our starting point for the analysis, Eq. (1), only describes the $K$ point of graphene. To capture intervalley scattering, one should also simultaneously include the $K^{\prime}$ point [54], which is accomplished by making the substitution $\boldsymbol{\sigma} \rightarrow \boldsymbol{\sigma}^{*}$ in Eq. (1) [1]. This can be considered as a next step in future work.

To model the confinement of massless Dirac fermions in a nanostructure, a "mass term" $\Delta(\boldsymbol{r})$ is added to Eq. (1):

$$
H_{0}=v_{F} \boldsymbol{p} \cdot \boldsymbol{\sigma}+\Delta(\boldsymbol{r}) \sigma_{z}
$$

where the function $\Delta(\boldsymbol{r})$ is defined at each point $\boldsymbol{r}$ in space and takes the value zero inside and $\Delta_{0}$ outside of the material. The goal is to simulate a "hard wall" barrier in the limit where $\Delta_{0} \rightarrow \infty$. In practice, setting $\Delta_{0}=170 \mathrm{eV}$ suffices [20].

A previous study 20] compared the simple, continuum Dirac Hamiltonian approach outlined here to a more refined description based on a tight-binding model which, in particular, can account for the distinct edge terminations (armchair, zigzag, etc.) of the graphene lattice. The authors found that minimizing the lengths of the zigzag edges around the holes reduces the presence of localized edge states, improving quantitative agreement between the two models. From a different perspective, one expects that edge effects will play a minor role in the overall properties of the system provided that dangling bonds are hydrogen passivated and edge spins are scrambled at ambient temperature. These points justify using a continuum Dirac Hamiltonian approach.

In this work, we focus on a representative triangular antidot lattice with circular (Fig.11a) or triangular holes (Fig.2a). The time-independent Schrödinger equation is

$$
H_{0} \varphi_{n \boldsymbol{k}}(\boldsymbol{r})=E_{n \boldsymbol{k}} \varphi_{n \boldsymbol{k}}(\boldsymbol{r}),
$$

where $n$ is the band index and $\hbar \boldsymbol{k}$ is the crystal momentum. Its solutions can be written in the Bloch form,

$$
\varphi_{n \boldsymbol{k}}(\boldsymbol{r})=e^{i \boldsymbol{k} \cdot \boldsymbol{r}} u_{n \boldsymbol{k}}(\boldsymbol{r}) .
$$

Thanks to its periodicity in space, $u_{n \boldsymbol{k}}(\boldsymbol{r})$ can be written as a Fourier series

$$
u_{n \boldsymbol{k}}(\boldsymbol{r})=\sum_{\boldsymbol{G}} u_{n \boldsymbol{k}}^{(\boldsymbol{G})} e^{i \boldsymbol{G} \cdot \boldsymbol{r}},
$$

where $\boldsymbol{G}=a \boldsymbol{G}_{1}+b \boldsymbol{G}_{2}, \boldsymbol{G}_{1}$ and $\boldsymbol{G}_{2}$ are the supercell reciprocal lattice vectors, and $a$ and $b$ are integers. The equation to solve is then

$$
\sum_{\boldsymbol{G}^{\prime}} \mathcal{H}_{\boldsymbol{k}}^{\left(\boldsymbol{G}, \boldsymbol{G}^{\prime}\right)} u_{n \boldsymbol{k}}^{\left(\boldsymbol{G}^{\prime}\right)}=E_{n \boldsymbol{k}} u_{n \boldsymbol{k}}^{(\boldsymbol{G})}
$$

where

$$
\mathcal{H}_{\boldsymbol{k}}^{\left(\boldsymbol{G}, \boldsymbol{G}^{\prime}\right)}=\left[\begin{array}{cc}
\Delta_{\boldsymbol{G}-\boldsymbol{G}^{\prime}} & T_{\boldsymbol{k} \boldsymbol{G}} \delta_{\boldsymbol{G}, \boldsymbol{G}^{\prime}} \\
T_{\boldsymbol{k} \boldsymbol{G}}^{*} \delta_{\boldsymbol{G}, \boldsymbol{G}^{\prime}} & -\Delta_{\boldsymbol{G}-\boldsymbol{G}^{\prime}}
\end{array}\right]
$$

with

$$
\Delta_{\boldsymbol{G}-\boldsymbol{G}^{\prime}} \equiv \frac{1}{A_{\mathrm{SC}}} \int d^{2} r \Delta(\boldsymbol{r}) e^{-i\left(\boldsymbol{G}-\boldsymbol{G}^{\prime}\right) \cdot \boldsymbol{r}}
$$

and

$$
T_{k G} \equiv \hbar v_{F}\left[\left(k_{x}+G_{x}\right)-i\left(k_{y}+G_{y}\right)\right] .
$$

The integration in Eq. (8) is over the supercell of area $A_{\mathrm{SC}}$. In principle, Eq. (6) is an infinite-dimensional matrix equation for each fixed $\boldsymbol{k}$ and $n$. In practice, by forming a block matrix $\mathcal{H}_{\boldsymbol{k}}$ using all combinations of $\boldsymbol{G}$ and $\boldsymbol{G}^{\prime}$ such that $a, b, a^{\prime}, b^{\prime} \in\left[-N_{\text {rec }}, N_{\text {rec }}\right]$ in Eq. (7), Eq. (6) can be rewritten as a finite matrix diagonalization problem:

$$
\mathcal{H}_{\boldsymbol{k}} \boldsymbol{u}_{n \boldsymbol{k}}=E_{n \boldsymbol{k}} \boldsymbol{u}_{n \boldsymbol{k}}
$$

where the components of $\boldsymbol{u}_{n \boldsymbol{k}}$ contain all of the $u_{n \boldsymbol{k}}^{(\boldsymbol{G})}$ in the allowed range. They are then used to construct approximate $u_{n \boldsymbol{k}}(\boldsymbol{r})$ (Eq. (5)) and, finally, the $\varphi_{n \boldsymbol{k}}(\boldsymbol{r})$ (Eq. (4)). For numerical evaluation in the model system under study, truncation at $N_{\text {rec }}=16$ spatial Fourier modes converges the electronic band structures $E_{n \boldsymbol{k}}$.

\section{FLOQUET GRAPHENE ANTIDOT LATTICES}

To investigate the effect of the applied control field, we rely on the standard prescription for minimally coupling the electromagnetic field to the Dirac Hamiltonian 
of Eq. (2). The result is the time-dependent Hamiltonian

$$
H(t)=H_{0}(\boldsymbol{p} \rightarrow \boldsymbol{p}+|e| \boldsymbol{A}(t))=H_{0}+|e| v_{F} \boldsymbol{A}(t) \cdot \boldsymbol{\sigma},
$$

where the vector potential $\boldsymbol{A}$ corresponds to a homogeneous, in-plane electric field $\boldsymbol{E}=-\partial_{t} \boldsymbol{A}$ with circular polarization, that is,

$$
\boldsymbol{A}(t)=\frac{E_{0}}{\Omega}[\cos (\Omega t), \sin (\Omega t), 0] .
$$

Thus, the control parameters are the electric field amplitude $E_{0}$ and the angular frequency $\Omega$. The vector potential of Eq. 12 misrepresents the magnetic flux density $(\boldsymbol{B}=\nabla \times \boldsymbol{A}=\mathbf{0})$. Nonetheless, it is the typical starting point for many other investigations of radiation driven graphene and we will follow this practice 33 51, 55, 61]. One can roughly assess the impact of ignoring the magnetic field by noting that the ratio of the magnitude of the magnetic to the electric force is at worst $v_{F} / c \approx 0.003$. In addition, the $\Omega t-\boldsymbol{K} \cdot \boldsymbol{r}$ that would normally appear as the argument of the cosine and sine functions in Eq. (12) reduces to $\Omega t$ since the relevant wave-vector can be chosen to be $\boldsymbol{K}=\left[0,0, K_{z}\right]$ and the graphene layer can be placed in the $z=0$ plane.

The next step is to move to the matrix representation of the time-dependent Hamiltonian $H(t)$ (Eq. (11)), in the basis obtained from solving the time-independent problem, recall Eq. (3). The matrix elements are

$H_{n n^{\prime} ; \boldsymbol{k}}(t)=E_{n \boldsymbol{k}} \delta_{n n^{\prime}}+|e| v_{F} \boldsymbol{A}(t) \cdot \int d^{2} r \varphi_{n \boldsymbol{k}}^{\dagger}(\boldsymbol{r}) \boldsymbol{\sigma} \varphi_{n^{\prime} \boldsymbol{k}}(\boldsymbol{r})$.

The driving renormalizes the individual bands and also generates inter-band coupling. For simplicity we focus on the two band model that emerges from keeping only the first band below and first band above the Fermi energy $(0 \mathrm{eV})$. From here forward, $H_{\boldsymbol{k}}(t)$ refers to the timedependent Hamiltonian in its matrix representation.

Since $H_{\boldsymbol{k}}(t)$ is periodic in time with period $T=2 \pi / \Omega$, the solution of the time-dependent Schrödinger equation,

$$
i \hbar \partial_{t} \psi_{n \boldsymbol{k}}(t)=H_{\boldsymbol{k}}(t) \psi_{n \boldsymbol{k}}(t),
$$

can be constructed using the extended space formulation of the Floquet formalism. Then the above time-dependent problem is formally mapped to a timeindependent problem of diagonalizing an associated Hermitian operator defined on an enlarged space (compared to the original, physical Hilbert space) [62]. The solutions take the form

$$
\psi_{n \boldsymbol{k}}(t)=e^{-i \epsilon_{n \boldsymbol{k}} t / \hbar} \Phi_{n \boldsymbol{k}}(t), \quad \Phi_{n \boldsymbol{k}}(t+T)=\Phi_{n \boldsymbol{k}}(t) .
$$

The periodicity of $H_{\boldsymbol{k}}(t)$ and $\Phi_{n \boldsymbol{k}}(t)$ allows for the Fourier decompositions in terms of time harmonics,

$$
H_{\boldsymbol{k}}(t)=\sum_{m} H_{\boldsymbol{k}}^{(m)} e^{-i m \Omega t}
$$

$$
\Phi_{n \boldsymbol{k}}(t)=\sum_{m} \phi_{n \boldsymbol{k}}^{(m)} e^{-i m \Omega t}
$$

where $m \in \mathbb{Z}$ is the temporal Fourier index. Eq. 14 can then be rewritten as

$$
\sum_{m^{\prime}} \tilde{H}_{\boldsymbol{k}}^{\left(m, m^{\prime}\right)} \phi_{n \boldsymbol{k}}^{\left(m^{\prime}\right)}=\epsilon_{n \boldsymbol{k}} \phi_{n \boldsymbol{k}}^{(m)}
$$

in terms of

$$
\begin{array}{r}
\tilde{H}_{\boldsymbol{k}}^{\left(m, m^{\prime}\right)}=\frac{1}{T} \int_{0}^{T} d t H_{\boldsymbol{k}}(t) e^{i\left(m-m^{\prime}\right) \Omega t}-\delta_{m m^{\prime}} m \hbar \Omega \mathbf{1} \\
=H_{\boldsymbol{k}}^{\left(m-m^{\prime}\right)}-\delta_{m m^{\prime}} m \hbar \Omega \mathbf{1} .
\end{array}
$$

Due to the simple form of the vector potential of Eq. (12), only matrix blocks with $\left|m-m^{\prime}\right| \leq 1$ can be non-zero. By forming a block matrix $H_{\boldsymbol{k}}^{\text {(Floquet) }}$ using all values of $m, m^{\prime}$ between $-N_{\text {Floquet }}$ and $N_{\text {Floquet }}$ in Eq. (19), Eq. 18 can then be rewritten as a matrix diagonalization problem

$$
H_{\boldsymbol{k}}^{(\text {Floquet })} \phi_{n \boldsymbol{k}}=\epsilon_{n \boldsymbol{k}} \boldsymbol{\phi}_{n \boldsymbol{k}} .
$$

For all cases to be considered below, truncation at $N_{\text {Floquet }}=5$ ensures converged quasienergy spectra $\epsilon_{n \boldsymbol{k}}$.

\section{FLOQUET BAND ENGINEERING IN GRAPHENE ANTIDOT LATTICES}

\section{IV.1. Static system}

As a point of reference, we first calculate the electronic band structures $E_{n \boldsymbol{k}}$ (recall Eq. (3p)), prior to irradiation, of several graphene antidot lattices. We find that, regardless of the shape of the hole, increasing the hole size while keeping the size of the supercell fixed changes the band structure from the characteristic gapless Dirac dispersion of the empty lattice (no holes) into flat bands for holes that are close to touching. Specifically, we choose the geometric parameters $L$ and $D$, see Fig.1 1 , or $L$ and $s$, see Fig. 2a, so that they are consistent with the physical characteristics of graphene. The parameter $L$ should only be on the order of a few nanometers for quantum confinement effects to play a role. In particular, we investigate circular holes of diameter $D=1.6 \mathrm{~nm}$ and $D=3.7 \mathrm{~nm}$, and equilateral triangular holes of side lengths $s=1.4 \mathrm{~nm}$ and $s=3.2 \mathrm{~nm}$. In all cases, $L=3.0$ $\mathrm{nm}$. The corresponding band structures are plotted in Figs.11b, 1k, 2b, and 2k. Quantum confinement opens a large band gap and adds curvature to the bands at the $\Gamma$ point of the Brillouin zone. We note that the continuum model remains valid on these length scales [20] and that atomically precise graphene antidot lattices are within the reach of current fabrication capabilities [63. 
(a)

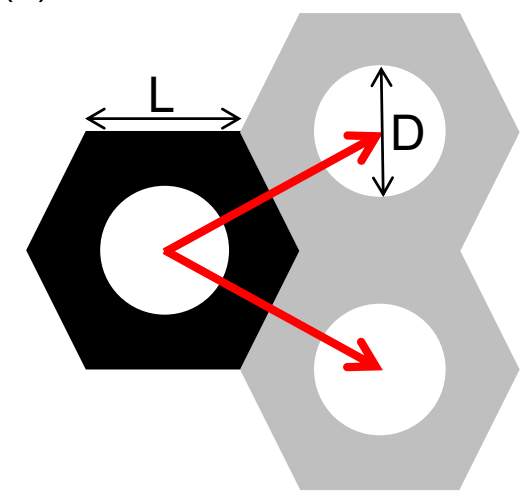

(b)

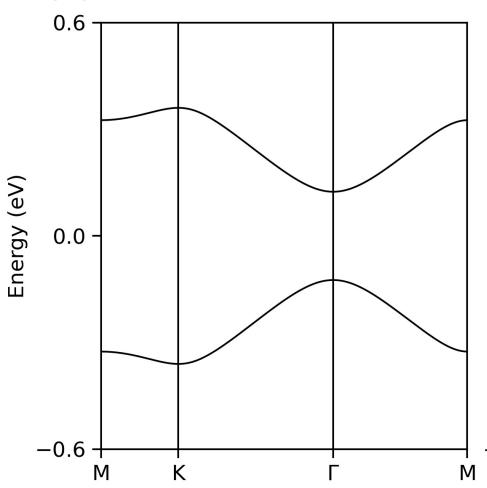

(c)

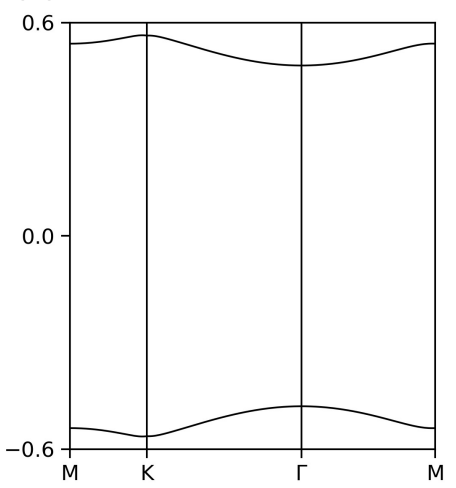

FIG. 1. (Color online) Triangular graphene antidot lattices with circular holes. (a) Geometric schematic with the superlattice vectors shown in red. The carbon lattice is not shown since we work within a continuum approach. Electronic band structure for $L=3.0 \mathrm{~nm}$ with (b) $D=1.6 \mathrm{~nm}$ and (c) $D=3.7 \mathrm{~nm}$. The Fermi energy is located at $0 \mathrm{eV}$.

(a)

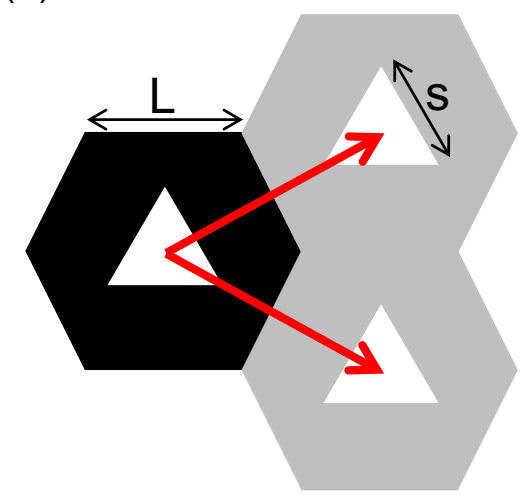

(b)

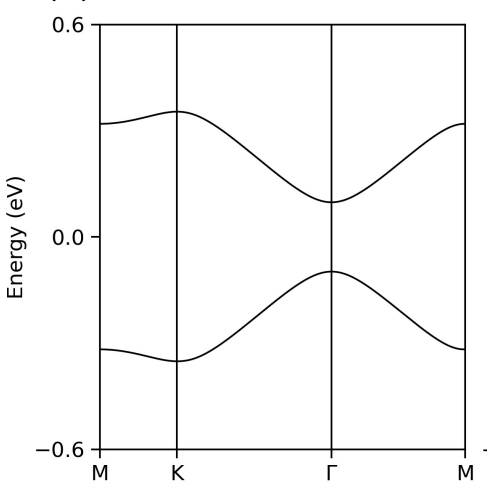

(c)

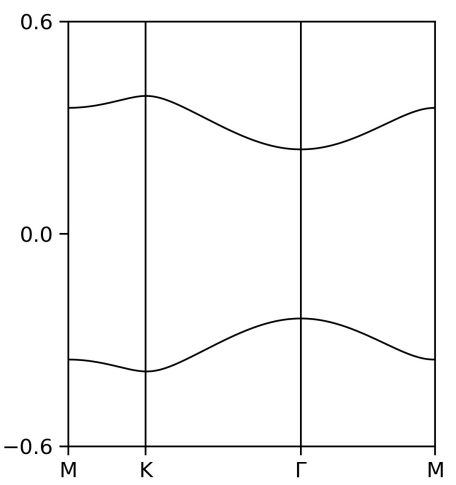

FIG. 2. (Color online) Triangular graphene antidot lattices with triangular holes. (a) Geometric schematic with the superlattice vectors shown in red. The carbon lattice is not shown since we work within a continuum approach. Electronic band structure for $L=3.0 \mathrm{~nm}$ with (b) $s=1.4 \mathrm{~nm}$ and (c) $s=3.2 \mathrm{~nm}$. The Fermi energy is located at $0 \mathrm{eV}$.

\section{IV.2. Floquet-driven system}

With the geometric parameters specified and the timeindependent problem solved, we are now well positioned to describe the periodically driven system. We choose the value of the photon energy $\hbar \Omega$ based on two criteria:

(i) The two static energy bands should automatically fall in the first Floquet Brillouin zone, that is, between $\pm \hbar \Omega / 2$. Under such a condition, there is a direct mapping between the zero electric field amplitude limit of the quasienergy spectrum $\epsilon_{n \boldsymbol{k}}$ and the electronic band structure $E_{n \boldsymbol{k}}$, as confirmed by our numerical simulations.

(ii) In order to justify the restriction to a two band model, the photon energy should not be high enough to trigger transitions from the first valence band to the second conduction band (not shown in our figures). For the structures described in Figs. 1 and 2 the appropriate photon energy ranges are

- $0.72-0.81 \mathrm{eV}$ (Fig.1p),
- 1.13-1.15 eV (Fig.11.),

- $0.71-0.77 \mathrm{eV}$ (Fig.2p),

- $0.78-0.85 \mathrm{eV}$ (Fig.22).

To ensure that our results are stable against small variations of the parameters, we calculate the quasienergy spectra for two values of $\hbar \Omega$ in these ranges of validity. The specific values of the photon energy are

- $0.75,0.80 \mathrm{eV}$ (Fig.1p),

- $1.13,1.15 \mathrm{eV}$ (Fig.11. $)$,

- $0.71,0.77 \mathrm{eV}$ (Fig.2p),

- $0.78,0.85 \mathrm{eV}$ (Fig.2 2).

In addition to the frequency of the radiation, one can also control the intensity. We scanned the electric field amplitude $E_{0}$ from 0 to 6 a.u. $(1 \mathrm{a} . \mathrm{u} .=1.291$ $(\mathrm{GeV} / \mathrm{nm}) / \mathrm{nC})$ in increments of 0.05 a.u., expanding and interpolating further when necessary. The units of $E_{0}$ are 


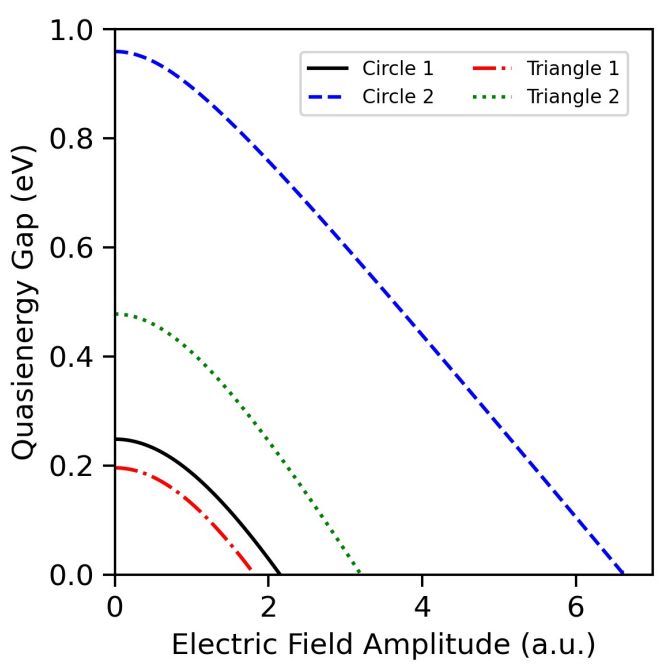

FIG. 3. (Color online) Continuous tuning of the Floquet quasienergy gap (at $\Gamma$ ) with the electric field amplitude. Each curve starts at the equilibrium state $(0$ a.u. $)$ and ends at the Floquet Dirac phase (horizontal axis intersection). Circle 1, Circle 2, Triangle 1, and Triangle 2 correspond to the structures defined in Figs. 1p, 11, 2p, and 2r.

taken from a previous paper on Floquet Dirac materials 64. The values of $E_{0}$ that we investigate are all well below the pulsed damage threshold for graphene, which is 23 a.u. [65]. This said, we find that the qualitative features of the quasienergy bands are the same for all cases after a rescaling of the electric field amplitude. As a quantitative illustration of this fact, we show in Fig. 3 the dependence of the quasienergy gap upon the electric field amplitude for our four Floquet graphene antidot lattices. Based on these results, for succinctness we only explicitly show and discuss the results for circular holes with $D=1.6 \mathrm{~nm}$ and $\hbar \Omega=0.8 \mathrm{eV}$ in what follows.

The insensitivity of the quasienergy spectra to the shape of the holes was an unexpected outcome of our simulations. A possible explanation is that the effective Dirac Hamiltonian only captures quantum confinement effects while softening or removing altogether the effect of particular edge configurations. The antidot lattice imposes complicated boundary conditions on the wave functions, see Eq. (3). This added layer of richness emerges in the quasienergy spectra via the matrix elements in Eq. (13). The particle-hole symmetry of the starting Dirac Hamiltonian (Eq. (1)) is preserved in all cases.

The quasienergy bands depicted in Fig. 4 summarize our main findings about the Floquet phases that the system can access as the electric field amplitude is varied in the parameter regime under exploration. Recall that, in the absence of driving, the Dirac point of pristine graphene is replaced by parabolic bands separated by a gap at the $\Gamma$ point for the graphene antidot lattice (see Fig. (4). Upon adding driving, a number of distinctive physical features emerge:
1. The Dirac point can be restored dynamically for a suitable value of the electric field amplitude that we call $E_{0 c_{1}}$. The quasienergy gap closes at the $\Gamma$ point of the Brillouin zone.

2. For $E_{0}>E_{0 c_{1}}$, the quasienergy gap reopens and moves to the $M$ point of the Brillouin zone; the bands flatten substantially close to the $\Gamma$ point.

3. There is a second value of the intensity $E_{0 c_{2}}>E_{0 c_{1}}$ for which the quasienergy gap closes again but now at the $M$ point. Moreover, the bands touch in a most peculiar way: they feature linear dispersion in one direction and quadratic in another.

4. The quasienergy gap reopens for $E_{0}>E_{0 c_{2}}$.

Thinking of the dynamical restoration of the Dirac point in the Floquet graphene antidot lattice as a phase transition, we shall call the phase with $0 \leq E_{0}<E_{0 c_{1}}$ the Floquet quasi-equilibrium phase and the phase with $E_{0 c_{1}}<E_{0}<E_{0 c_{2}}$, which ends with the closing of the quasienergy gap at $M$, the first Floquet phase, or Floquet Dirac phase. There is a second Floquet phase, or Floquet semi-Dirac phase, for $E_{0}>E_{0 c_{2}}$. It is worth it to further expand on these remarkable features and speculate on how they physically come about.

1.: The ability to restore the Dirac point (Fig.4 4c) seems only possible because the "mass term" in the Hamiltonian of Eq. (2) hides the Dirac dispersion of standard graphene, and is then dynamically canceled by the radiation field. While pristine graphene already features a Dirac point, having the non-driven gapped bands and the Floquet Dirac point enables at least two novel applications, as we argue in Sec.V. Furthermore, varying the geometric parameters of static graphene antidot lattices only results in discrete tunability of the electronic band gap. By contrast, the electric field amplitude can be varied continuously, which leads to an adiabatic connection between the static state and the Floquet Dirac regime. In Fig. 3 we explicitly see how the quasienergy gap, which remains at $\Gamma$, can be tuned continuously and monotonically.

2.: Non-ballistic transport, the phonon-assisted decays that relax excited carriers, and the lifetimes of the states involved in optical transitions all depend on the nature of the electron-phonon coupling. This, in turn, depends on the high-symmetry point where the band extrema occur [66]. Therefore, the ability to shift the quasienergy gap from the $\Gamma$ point in the equilibrium phase to the $M$ point in the Floquet phase (Fig. $4 \mathrm{~d}$ ) can affect these three fundamental processes significantly via the difference in electron-phonon coupling between high-symmetry points. These phenomena could be investigated in quantitative detail with an adapted Floquet-Boltzmann approach 67.

A next notable feature is localization, which manifests as band flattening. On the one hand, in the absence of driving, the bands are flattened if the holes of an antidot lattice are forced to nearly touch. This flattening is 
(a)
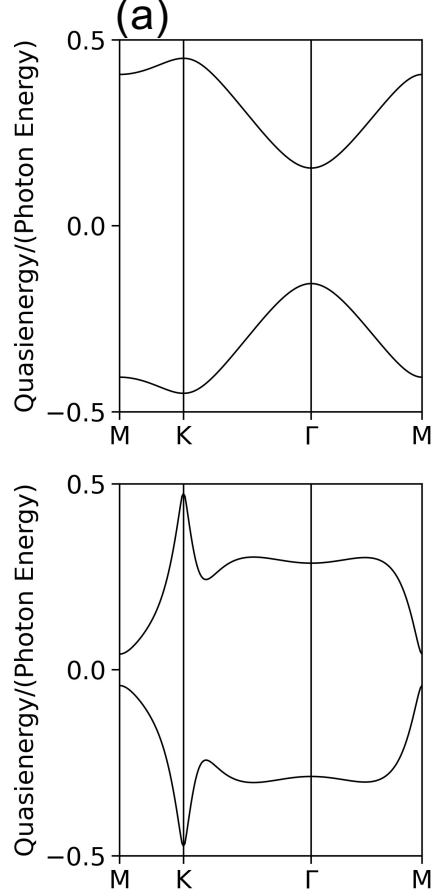

(e)
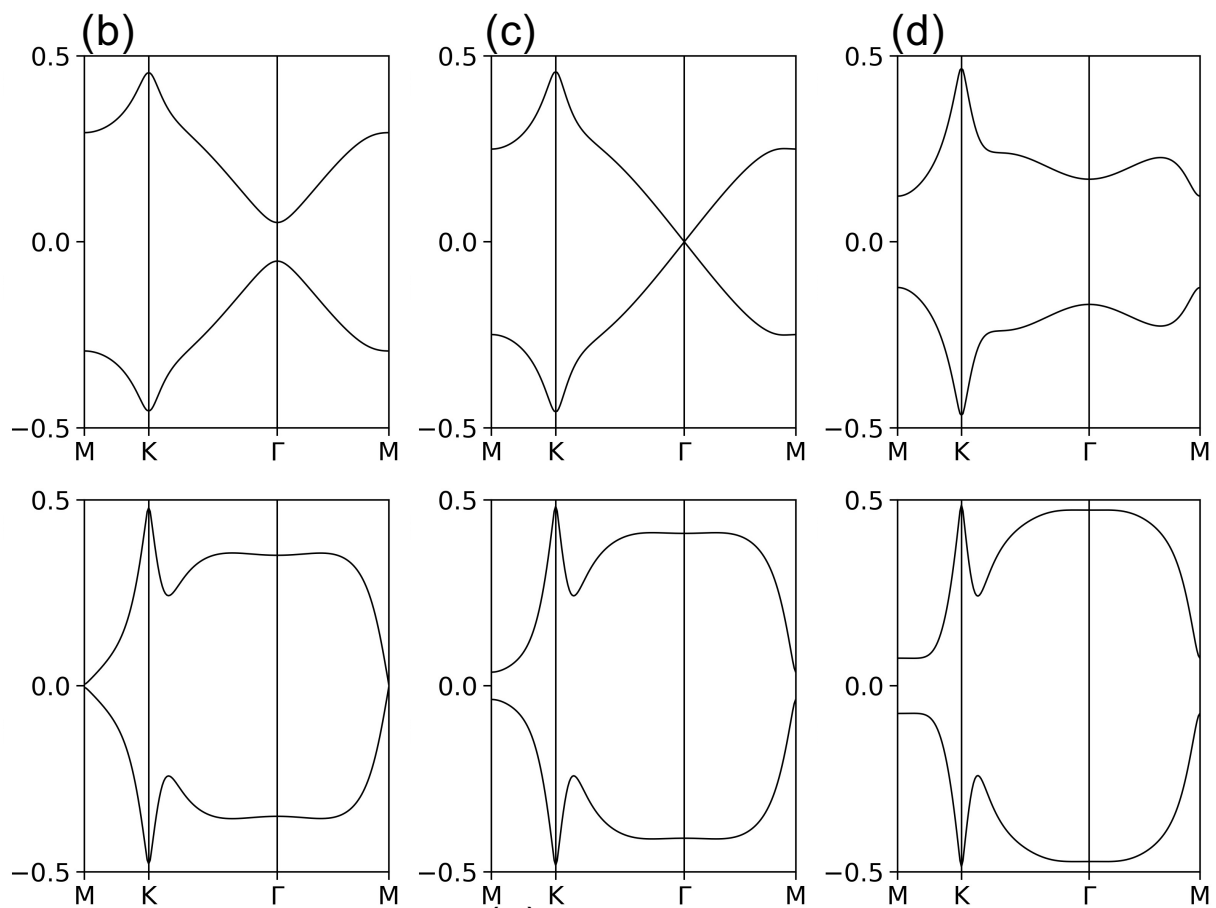

(f)

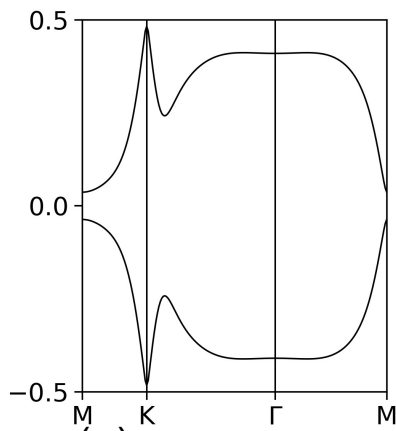

(g)

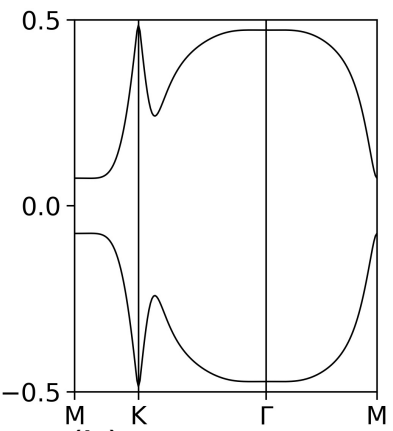

(h)

FIG. 4. Representative quasienergy spectra for the graphene antidot lattice defined in Fig. 1p, irradiated with circularly polarized light at a photon energy of $0.8 \mathrm{eV} . E_{0}$ is the electric field amplitude, where 1 a.u. $=1.291(\mathrm{GeV} / \mathrm{nm}) / \mathrm{nC}[64$. (a) $E_{0}=0$ a.u. Zero electric field amplitude limit for reference. (b) $E_{0}=1.7$ a.u. (c) $E_{0}=E_{0 c_{1}}=2.15$ a.u. The Dirac dispersion of standard graphene is effectively restored. (d) $E_{0}=3.4$ a.u. The quasienergy gap is shifted to the $M$ point. (e) $E_{0}=4.2$ a.u. The quasienergy bands are selectively flattened in reciprocal space near the $\Gamma$ point. (f) $E_{0}=E_{0 c_{2}}=4.62$ a.u. In moving from $K$ to $M$ the bands are quadratic, whereas in moving from $\Gamma$ to $M$ they are linear, indicative of a Floquet semi-Dirac material. (g) $E_{0}=5$ a.u. (h) $E_{0}=5.4$ a.u.

non-selective, in the sense that it occurs over the entire high-symmetry lines. On the other hand, our periodically driven system with $E_{0}=4.2$ a.u. (Fig.4 4 ) features bands which are selectively flattened near $\Gamma$ in reciprocal space. On the basis of the Hellmann-Feynman theorem, the group velocity of the quasienergy bands is given by $\boldsymbol{v}_{n \boldsymbol{k}}=\hbar^{-1} \nabla_{\boldsymbol{k}} \epsilon_{n \boldsymbol{k}}$ [68. Therefore, near the $\Gamma$ point, the charge carriers have zero group velocity, which is indicative of selective dynamical localization. This behavior persists as the electric field amplitude continues to increase, at least in the range of values considered here. Furthermore, since the band flattening occurs near $\Gamma$ and not another high-symmetry point, the system acts as a low-wavelength-pass electronic filter. For comparison, let us briefly mention here previous work [69, showing that periodic kicking of standard graphene can result in complete dynamical localization along one entire axis in reciprocal space or over the whole Brillouin zone.

3.: In Fig.4f, quadratic and linear dispersions emerge from the $M$ point in orthogonal directions, which correspond to "non-relativistic-like" and "relativistic-like" carriers, respectively. This behavior is the signature of a Floquet semi-Dirac material, which has already been observed for standard Floquet graphene 60, 61, 69. Even so, the physical mechanism of this striking anisotropy is not the same for all cases. In previous work, it was linearly polarized radiation [60, 61] or uniaxial periodic kicking 69 which generated the inequivalence between different directions in reciprocal space. In our setup, since we apply circularly polarized radiation, the anisotropy is a truly emergent phenomenon. In fact, in a different context, this is reminiscent of the anisotropic quasiparticle gap closing that has been found to emerge from competing interactions in both static and Floquetdriven gapless $s$-wave superconductors on 2D square lattices 70, 71.

As a platform for realizing a Floquet semi-Dirac material, Floquet graphene antidot lattices offer some distinct advantages over existing approaches. First, the semiDirac property can be realized at much lower photon energies $(\hbar \Omega=0.72-0.81 \mathrm{eV})$, whereas previously values of around at least $4 \mathrm{eV}$ were required [60,61. Second, while the work on periodic kicking is of fundamental physical interest, the proposals to realize it experimentally are not practical at the time of writing 69. Third, in the case of pristine graphene, the static Dirac and the Floquet semiDirac points are both gapless. On the other hand, for the graphene antidot lattice, the static system is gapped and the Floquet semi-Dirac point is gapless. Having a different quasienergy gap between the static and Floquet 
states may enable different applications.

4.: The first Floquet phase ends with the closing of the quasienergy gap at the $M$ point. The gap reopens immediately for $E_{0}>E_{0 c_{2}}$, landing one in a second Floquet phase. We show a couple of quasienergy band structures for this phase in Figs. $4 \mathrm{~s}$ and $4 \mathrm{~h}$. There are several other Floquet phases beyond this second one and below the damage threshold of $E_{0}=23$ a.u. We leave them as a topic for future research.

\section{APPLICATIONS}

As we showed in the previous section, the irradiated graphene antidot lattice can cycle between gapped, quadratic bands for vanishing intensity and a dynamically induced Dirac point for a suitable value of the intensity. We recognize at least two possible applications of this behavior. In this paper we will give only a qualitative description of them, leaving a more quantitative study for the future. At this level of analysis, we envision that the operation timescale of any such Floquet device should be much slower than the drive period, so that the initial state does not change appreciably within a period and a description in terms of the Floquet stroboscopic dynamics will suffice 62. In addition, and this point is specific to our setup, we do not know exactly what to expect for the mobility of the charge carriers at the dynamically induced Dirac point. We judge that a more quantitative description of our devices will have to be grounded in simulations within the Floquet LandauerBüttiker formalism [72, 73].

\section{V.1. An optoelectronic switch}

The first application we envision is an optoelectronic switch, which could function as an optical computing element. For this device, a steady voltage drop is held across the graphene antidot lattice during operation. Hence, without driving, the gapped system should support a small current which corresponds to the "off" state of the switch. By contrast, with suitable driving, the gapless Floquet phase with the Dirac dispersion should allow for a much larger current corresponding to the "on" state. Notice that the operating principle of this optoelectronic switch goes beyond the photoconductivity level (meaning that photons promote additional carriers into the conduction band). Rather, the operation mechanism is the renormalization of the band structure by the driving. Future numerical simulations should characterize the device more precisely through the on/off current ratio.

\section{V.2. A dynamically tunable electronic waveguide}

The second application we envision is a device that could be described as a dynamically tunable electronic waveguide. If the antidot lattice is only irradiated in a particular region of space, then one expects that the charge carriers will preferentially follow the path where the Dirac dispersion is restored. This idea presupposes that the properties we discovered still hold when translational symmetry is broken on the length scales of the waveguide. Our waveguide proposal is inspired by previous work where a graphene antidot lattice waveguide was formed by selectively leaving the graphene unpatterned, thus locally restoring the Dirac dispersion of standard graphene and creating a current channel 74. However, in that case the waveguide path was fixed, whereas in our proposal we conjecture that spatially selective irradiation should permit dynamical control of the current channels.

\section{CONCLUSIONS AND OUTLOOK}

We introduce the Floquet graphene antidot lattice as a platform for investigating the properties of quantum confined and periodically driven massless Dirac fermions in $2 \mathrm{D}$. The actual system is a sheet of graphene decorated by a periodic array of holes (the "antidots") and subjected to steady irradiation. To focus on the physics of massless Dirac fermions, we model the graphene antidot lattice in terms of a Dirac Hamiltonian with a spatially varying "mass term." Time-periodic driving is introduced by including an electromagnetic field at the minimal coupling level. We use the extended space formulation of the Floquet formalism to compute (numerically) the quasienergy band structures.

A number of notable properties emerge for various electric field amplitudes. First, the Dirac point, which had been removed by the antidot lattice, can be dynamically restored by irradiation. Second, the quasienergy gap can be shifted from one high-symmetry point of the Brillouin zone, the $\Gamma$ point, to another, the $M$ point, and that shift could affect dramatically the electron-phonon coupling in the system. Third, after the gap has shifted, the bands can be flattened near the $\Gamma$ point, indicative of selective dynamical localization. Finally, the quasienergy gap can again close at the $M$ point accompanied by exotic semiDirac behavior, where orthogonal directions emanating from the $M$ point feature quadratic and linear dispersions. Every one of these features is of intrinsic physical interest, but we have also pointed out potential device applications: an optoelectronic switch, a dynamically tunable electronic waveguide, and a low-wavelength-pass electronic filter. In general, the ability to cycle between different electronic phases with lasers may play an important role in the development of optoelectronic devices.

Some qualitative features of our quasienergy spectra can also be observed for systems different from ours, but we have made a careful case that the Floquet graphene antidot lattice may offer distinct practical advantages. From an experimental perspective, all of our predictions are valid for accessible near-IR radiation, in contrast to standard graphene, where the same non-resonant above 
bandwidth limit corresponds to ionizing extreme UV photon energies. Moreover, while we focused on graphene antidot lattices, everything was based on effective Dirac Hamiltonians, where a rescaling of parameters can be used to translate our results to other 2D Dirac materials.

We conclude with some ideas for future research. One immediate question that we have not answered is whether the Floquet graphene antidot lattice features non-trivial topology [75] 77]. More precisely, this system combines two gapping mechanisms, the time-reversalsymmetry-breaking circularly polarized light and the chiral-symmetry-breaking antidot lattice, both of which retain the particle-hole (charge-conjugation) symmetry. We have not determined whether the resulting quasienergy gap is topologically non-trivial, or if such properties vary based on the defined phase regimes (quasi-equilibrium, Floquet Dirac, Floquet semi-Dirac).
Another interesting question is how irregularities in the placement and shapes of the holes (disorder) affect the emergent properties [78, 81]. And finally, there is the critical question that we have asked before: What is the mobility of charge carriers at the emergent Dirac point?

\section{ACKNOWLEDGEMENTS}

This work was supported by the NSF under grant No. OIA-1921199. The computations in this work were performed on the Discovery cluster and HPC environments supported by the Research Computing group, IT\&C at Dartmouth College. We thank Stephen Carr for a critical reading of this manuscript and Vincent P. Flynn for discussions about the thermal conductivity of periodically driven systems.
[1] A. H. Castro Neto, F. Guinea, N. M. R. Peres, K. S. Novoselov, and A. K. Geim, The electronic properties of graphene, Rev. Mod. Phys. 81, 109 (2009).

[2] M. Y. Han, B. Özyilmaz, Y. Zhang, and P. Kim, Energy band-gap engineering of graphene nanoribbons, Phys. Rev. Lett. 98, 206805 (2007).

[3] T. G. Pedersen, C. Flindt, J. Pedersen, N. A. Mortensen, A.-P. Jauho, and K. Pedersen, Graphene antidot lattices: Designed defects and spin qubits, Phys. Rev. Lett. 100, 136804 (2008).

[4] T. G. Pedersen, C. Flindt, J. Pedersen, A.-P. Jauho, N. A. Mortensen, and K. Pedersen, Optical properties of graphene antidot lattices, Phys. Rev. B 77, 245431 (2008).

[5] M. Vanević, V. M. Stojanović, and M. Kindermann, Character of electronic states in graphene antidot lattices: Flat bands and spatial localization, Phys. Rev. B 80, 045410 (2009).

[6] J. A. Fürst, T. G. Pedersen, M. Brandbyge, and A.-P. Jauho, Density functional study of graphene antidot lattices: Roles of geometrical relaxation and spin, Phys. Rev. B 80, 115117 (2009).

[7] R. Petersen and T. G. Pedersen, Quasiparticle properties of graphene antidot lattices, Phys. Rev. B 80, 113404 (2009).

[8] J. A. Fürst, J. G. Pedersen, C. Flindt, N. A. Mortensen, M. Brandbyge, T. G. Pedersen, and A.-P. Jauho, Electronic properties of graphene antidot lattices, New J. Phys. 11, 095020 (2009).

[9] W. Liu, Z. F. Wang, Q. W. Shi, J. Yang, and F. Liu, Band-gap scaling of graphene nanohole superlattices, Phys. Rev. B 80, 233405 (2009).

[10] R. Martinazzo, S. Casolo, and G. F. Tantardini, Symmetry-induced band-gap opening in graphene superlattices, Phys. Rev. B 81, 245420 (2010).

[11] F. Ouyang, Z. Yang, J. Xiao, D. Wu, and H. Xu, Electronic structure and chemical modification of graphene antidot lattices, J. Phys. Chem. C 114, 15578 (2010).

[12] R. Petersen, T. G. Pedersen, and A.-P. Jauho, Clar sextet analysis of triangular, rectangular, and honeycomb graphene antidot lattices, ACS Nano 5, 523 (2011).
[13] A. Zhang, H. F. Teoh, Z. Dai, Y. P. Feng, and C. Zhang, Band gap engineering in graphene and hexagonal BN antidot lattices: A first principles study, Appl. Phys. Lett. 98, 023105 (2011).

[14] F. Ouyang, S. Peng, Z. Liu, and Z. Liu, Bandgap opening in graphene antidot lattices: The missing half, ACS Nano 5, 4023 (2011).

[15] R. Petersen, T. G. Pedersen, and A.-P. Jauho, Clar sextets in square graphene antidot lattices, Physica E Low Dimens. Syst. Nanostruct. 44, 967 (2012).

[16] T. G. Pedersen and J. G. Pedersen, Transport in graphene antidot barriers and tunneling devices, J. Appl. Phys. 112, 113715 (2012).

[17] X. Liu, Z. Zhang, and W. Guo, Universal rule on chirality-dependent bandgaps in graphene antidot lattices, Small 9, 1405 (2013).

[18] F. Ouyang, Z. Yang, S. Peng, X. Zheng, and X. Xiong, Antidot-dependent bandgap and Clar sextets in graphene antidot lattices, Physica E Low Dimens. Syst. Nanostruct. 56, 222 (2014).

[19] M. L. Trolle, U. S. Møller, and T. G. Pedersen, Large and stable band gaps in spin-polarized graphene antidot lattices, Phys. Rev. B 88, 195418 (2013).

[20] S. J. Brun, M. R. Thomsen, and T. G. Pedersen, Electronic and optical properties of graphene antidot lattices: Comparison of Dirac and tight-binding models, J. Phys. Condens. Matter 26, 265301 (2014).

[21] S. J. Thomsen, M. R.and Brun and T. G. Pedersen, Dirac model of electronic transport in graphene antidot barriers, J. Phys. Condens. Matter 26, 335301 (2014).

[22] F. Ouyang, S. Peng, Y. Yang, Z.and Chen, H. Zou, and X. Xiong, Bandgap opening/closing of graphene antidot lattices with zigzag-edged hexagonal holes, Phys. Chem. Chem. Phys. 16, 20524 (2014).

[23] G. Tang, Z. Zhang, X. Deng, Z. Fan, Y. Zeng, and J. Zhou, Improved scaling rules for bandgaps in graphene nanomeshs, Carbon 76, 348 (2014).

[24] J. Bai, X. Zhong, S. Jiang, Y. Huang, and X. Duan, Graphene nanomesh, Nat. Nanotechnol. 5, 190 (2010).

[25] B. S. Jessen, L. Gammelgaard, M. R. Thomsen, D. M. A. Mackenzie, J. D. Thomsen, J. M. Caridad, E. Duegaard, 
K. Watanabe, T. Taniguchi, T. J. Booth, et al., Lithographic band structure engineering of graphene, Nat. Nanotechnol. 14, 340 (2019).

[26] M. Bukov, L. D'Alessio, and A. Polkovnikov, Universal high-frequency behavior of periodically driven systems: From dynamical stabilization to Floquet engineering, Adv. Phys. 64, 139 (2015).

[27] D. N. Basov, R. D. Averitt, and D. Hsieh, Towards properties on demand in quantum materials, Nat. Mater. 16, 1077 (2017).

[28] T. Oka and S. Kitamura, Floquet engineering of quantum materials, Annu. Rev. Condens. Matter Phys. 10, 387 (2019).

[29] U. De Giovannini and H. Hübener, Floquet analysis of excitations in materials, J. Phys. Mater. 3, 012001 (2020).

[30] F. Harper, R. Roy, M. S. Rudner, and S. L. Sondhi, Topology and broken symmetry in Floquet systems, Annu. Rev. Condens. Matter Phys. 11, 345 (2020).

[31] M. S. Rudner and N. H. Lindner, Band structure engineering and non-equilibrium dynamics in Floquet topological insulators, Nat. Rev. Phys. 2, 229 (2020).

[32] M. Rodriguez-Vega, M. Vogl, and G. A. Fiete, Lowfrequency and Moiré Floquet engineering: A review, Ann. Phys. , 168434 (2021).

[33] S. V. Syzranov, M. V. Fistul, and K. B. Efetov, Effect of radiation on transport in graphene, Phys. Rev. B 78, 045407 (2008).

[34] F. J. López-Rodríguez and G. G. Naumis, Analytic solution for electrons and holes in graphene under electromagnetic waves: Gap appearance and nonlinear effects, Phys. Rev. B 78, 201406(R) (2008).

[35] T. Oka and H. Aoki, Photovoltaic Hall effect in graphene, Phys. Rev. B 79, 081406(R) (2009).

[36] O. V. Kibis, Metal-insulator transition in graphene induced by circularly polarized photons, Phys. Rev. B 81, 165433 (2010).

[37] H. L. Calvo, H. M. Pastawski, S. Roche, and L. E. F. Foa Torres, Tuning laser-induced band gaps in graphene, Appl. Phys. Lett. 98, 232103 (2011).

[38] Y. Zhou and M. W. Wu, Optical response of graphene under intense terahertz fields, Phys. Rev. B 83, 245436 (2011).

[39] S. E. Savel'ev and A. S. Alexandrov, Massless Dirac fermions in a laser field as a counterpart of graphene superlattices, Phys. Rev. B 84, 035428 (2011).

[40] H. L. Calvo, P. M. Perez-Piskunow, S. Roche, and L. E. F. Foa Torres, Laser-induced effects on the electronic features of graphene nanoribbons, Appl. Phys. Lett. 101, 253506 (2012).

[41] H. L. Calvo, P. M. Perez-Piskunow, H. M. Pastawski, S. Roche, and L. E. F. Foa Torres, Non-perturbative effects of laser illumination on the electrical properties of graphene nanoribbons, J. Phys. Condens. Matter 25, 144202 (2013).

[42] Z. Gu, H. A. Fertig, D. P. Arovas, and A. Auerbach, Floquet spectrum and transport through an irradiated graphene ribbon, Phys. Rev. Lett. 107, 216601 (2011).

[43] P. M. Perez-Piskunow, G. Usaj, C. A. Balseiro, and L. E. F. Foa Torres, Floquet chiral edge states in graphene, Phys. Rev. B 89, 121401(R) (2014).

[44] X. Zhai and G. Jin, Photoinduced topological phase transition in epitaxial graphene, Phys. Rev. B 89, 235416 (2014).
[45] G. Usaj, P. M. Perez-Piskunow, L. E. F. Foa Torres, and C. A. Balseiro, Irradiated graphene as a tunable Floquet topological insulator, Phys. Rev. B 90, 115423 (2014).

[46] P. M. Perez-Piskunow, L. E. F. Foa Torres, and G. Usaj, Hierarchy of Floquet gaps and edge states for driven honeycomb lattices, Phys. Rev. A 91, 043625 (2015).

[47] M. Puviani, F. Manghi, and A. Bertoni, Dynamics and control of edge states in laser-driven graphene nanoribbons, Phys. Rev. B 95, 235430 (2017).

[48] W. Wang, X. Lü, and H. Xie, Floquet bands and photoninduced topological edge states of graphene nanoribbons, Chin. Phys. B 30, 066701 (2021).

[49] M. A. Sentef, M. Claassen, A. F. Kemper, B. Moritz, T. Oka, J. K. Freericks, and T. P. Devereaux, Theory of Floquet band formation and local pseudospin textures in pump-probe photoemission of graphene, Nat. Commun. 6, 1 (2015).

[50] M. Schüler, U. De Giovannini, H. Hübener, A. Rubio, M. A. Sentef, T. P. Devereaux, and P. Werner, How circular dichroism in time-and angle-resolved photoemission can be used to spectroscopically detect transient topological states in graphene, Phys. Rev. X 10, 041013 (2020).

[51] Y. Chen, Y. Wang, M. Claassen, B. Moritz, and T. P. Devereaux, Observing photo-induced chiral edge states of graphene nanoribbons in pump-probe spectroscopies, npj Quantum Mater. 5, 1 (2020).

[52] J. W. McIver, B. Schulte, F.-U. Stein, T. Matsuyama, G. Jotzu, G. Meier, and A. Cavalleri, Light-induced anomalous Hall effect in graphene, Nat. Phys. 16, 38 (2020).

[53] S. A. Sato, J. W. McIver, M. Nuske, P. Tang, G. Jotzu, B. Schulte, H. Hübener, U. De Giovannini, L. Mathey, M. A. Sentef, et al., Microscopic theory for the lightinduced anomalous Hall effect in graphene, Phys. Rev. B 99, 214302 (2019).

[54] J. N. B. Rodrigues, Intervalley scattering of graphene massless Dirac fermions at 3-periodic grain boundaries, Phys. Rev. B 94, 134201 (2016).

[55] G. E. Topp, G. Jotzu, J. W. McIver, L. Xian, A. Rubio, and M. A. Sentef, Topological Floquet engineering of twisted bilayer graphene, Phys. Rev. Research 1, 023031 (2019).

[56] Y. Li, H. A. Fertig, and B. Seradjeh, Floquet-engineered topological flat bands in irradiated twisted bilayer graphene, Phys. Rev. Research 2, 043275 (2020).

[57] M. Vogl, M. Rodriguez-Vega, and G. A. Fiete, Floquet engineering of interlayer couplings: Tuning the magic angle of twisted bilayer graphene at the exit of a waveguide, Phys. Rev. B 101, 241408(R) (2020).

[58] M. Vogl, M. Rodriguez-Vega, and G. A. Fiete, Effective Floquet Hamiltonians for periodically driven twisted bilayer graphene, Phys. Rev. B 101, 235411 (2020).

[59] D. A. Lovey, G. Usaj, L. E. F. Foa Torres, and C. A. Balseiro, Floquet bound states around defects and adatoms in graphene, Phys. Rev. B 93, 245434 (2016).

[60] P. Delplace, Á. Gómez-León, and G. Platero, Merging of Dirac points and Floquet topological transitions in ACdriven graphene, Phys. Rev. B 88, 245422 (2013).

[61] H. Liu, J.-T. Sun, and S. Meng, Engineering Dirac states in graphene: Coexisting type-I and type-II Floquet-Dirac fermions, Phys. Rev. B 99, 075121 (2019).

[62] M. S. Rudner and N. H. Lindner, The Floquet engineer's handbook, arXiv:2003.08252v2 (2020). 
63] C. Moreno, M. Vilas-Varela, B. Kretz, A. Garcia-Lekue, M. V. Costache, M. Paradinas, M. Panighel, G. Ceballos, S. O. Valenzuela, D. Peña, et al., Bottom-up synthesis of multifunctional nanoporous graphene, Science 360, 199 (2018).

[64] H. Hübener, M. A. Sentef, U. De Giovannini, A. F. Kemper, and A. Rubio, Creating stable Floquet-Weyl semimetals by laser-driving of 3D Dirac materials, Nat. Commun. 8, 1 (2017).

[65] A. Roberts, D. Cormode, C. Reynolds, T. NewhouseIllige, B. J. LeRoy, and A. S. Sandhu, Response of graphene to femtosecond high-intensity laser irradiation, Appl. Phys. Lett. 99, 051912 (2011).

[66] F. Giustino, Electron-phonon interactions from first principles, Rev. Mod. Phys. 89, 015003 (2017).

[67] M. Genske and A. Rosch, Floquet-Boltzmann equation for periodically driven Fermi systems, Phys. Rev. A 92 , 062108 (2015).

[68] H. Schanz, T. Dittrich, and R. Ketzmerick, Directed chaotic transport in Hamiltonian ratchets, Phys. Rev. E 71, 026228 (2005).

[69] A. Agarwala, U. Bhattacharya, A. Dutta, and D. Sen, Effects of periodic kicking on dispersion and wave packet dynamics in graphene, Phys. Rev. B 93, 174301 (2016).

[70] S. Deng, G. Ortiz, A. Poudel, and L. Viola, Majorana flat bands in $s$-wave gapless topological superconductors, Phys. Rev. B 89, 140507(R) (2014).

[71] A. Poudel, G. Ortiz, and L. Viola, Dynamical generation of Floquet Majorana flat bands in $s$-wave superconductors, EPL 110, 17004 (2015).
[72] S. Kohler, J. Lehmann, and P. Hänggi, Driven quantum transport on the nanoscale, Phys. Rep. 406, 379 (2005).

[73] M. Fruchart, P. Delplace, J. Weston, X. Waintal, and D. Carpentier, Probing (topological) Floquet states through DC transport, Physica E Low Dimens. Syst. Nanostruct. 75, 287 (2016).

[74] J. G. Pedersen, T. Gunst, T. Markussen, and T. G. Pedersen, Graphene antidot lattice waveguides, Phys. Rev. B 86, 245410 (2012).

[75] X. Zhang and M. Zhao, Prediction of quantum anomalous Hall effect on graphene nanomesh, RSC Adv. 5, 9875 (2015).

[76] J. Pan, T. Zhang, H. Zhang, B. Zhang, Z. Dong, and P. Sheng, Berry curvature and nonlocal transport characteristics of antidot graphene, Phys. Rev. X 7, 031043 (2017).

[77] T. Kariyado, Y.-C. Jiang, H. Yang, and X. Hu, Counterpropagating topological interface states in graphene patchwork structures with regular arrays of nanoholes, Phys. Rev. B 98, 195416 (2018).

[78] V. Hung Nguyen, M. Chung Nguyen, H.-V. Nguyen, and P. Dollfus, Disorder effects on electronic bandgap and transport in graphene-nanomesh-based structures, J. Appl. Phys. 113, 013702 (2013).

[79] S. Yuan, R. Roldán, A.-P. Jauho, and M. I. Katsnelson, Electronic properties of disordered graphene antidot lattices, Phys. Rev. B 87, 085430 (2013).

[80] S. R. Power and A.-P. Jauho, Electronic transport in disordered graphene antidot lattice devices, Phys. Rev. B 90, 115408 (2014).

[81] Z. Fan, A. Uppstu, and A. Harju, Electronic and transport properties in geometrically disordered graphene antidot lattices, Phys. Rev. B 91, 125434 (2015). 